\title{
Refined and lethal
}

BCL-2 and related family members such as BCL- $\mathrm{X}_{\mathrm{L}}$ are anti-apoptotic proteins that are being pharmacologically targeted to potentiate apoptosis as an anticancer strategy. However, achieving efficacy while minimizing unwanted toxicity has remained a challenge. Two new studies have reported improvements in the effectiveness of these agents by developing a more selective drug and by devising a rational combination strategy.

So far, BCL-2-targeted agents such as navitoclax (ABT-263) have shown most promise in lymphomas and lymphocytic leukaemias owing to the frequent overexpression of BCL-2 that is caused by various genetic lesions. The doses of navitoclax that can be used clinically have been limited by the accompanying thrombocytopenia,

tumour regression in an orthotopic mouse model which is caused by the inhibition of BCL- $\mathrm{X}_{\mathrm{L}}$ in platelets. To address this problem, Andrew Souers and colleagues used co-crystal structures of BCL-2 bound to inhibitors to design a more selective navitoclax derivative, ABT-199. Unlike the similar affinities

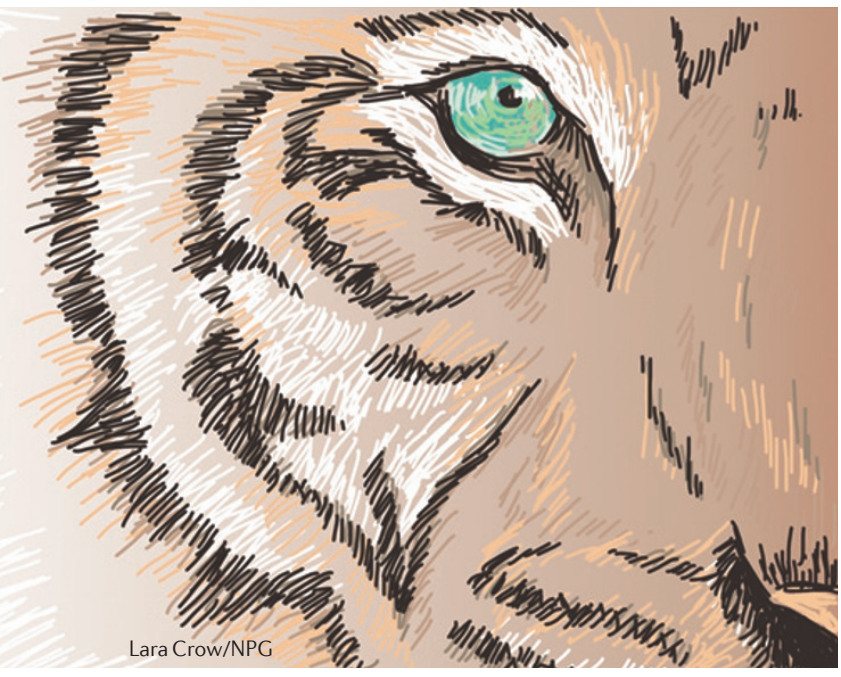

of navitoclax for BCL-2 and BCL-X ABT-199 showed $>1,000$-fold selectivity for BCL-2.

ABT-199 induced apoptosis in a subset of lymphoma and lymphocytic leukaemia cell lines treated in vitro, at similar or lower doses to navitoclax. As expected, cells expressing high BCL-2 levels were most sensitive, and ABT-199 was shown to function by blocking complex formation between BCL-2 and pro-apoptotic binding partners such as BIM. Crucially, ABT-199 had substantially less cytotoxicity than navitoclax towards BCL- $\mathrm{X}_{\mathrm{L}}$-dependent cell lines in vitro and human platelets ex vivo.

In mouse xenografts of human cell lines that are known to respond to BCL-2 inhibition, ABT-199 treatment resulted in a reduction in tumour growth without accompanying toxicity. Furthermore, combining ABT-199 with the B-cell-targeted monoclonal antibody rituximab and the chemotherapeutic agent bendamustine enhanced the growth suppression of B cell lymphoma xenografts, implying that combination approaches are worthy of further investigation.

ABT-199 was also tested in three patients with chronic lymphocytic leukaemia who had become refractory to prior standard treatments. Within 24 hours, ABT-199 as a single agent substantially reduced circulating lymphocyte counts in the two patients that had pretreatment lymphocytosis, and it had only modest effects on circulating platelet numbers in all three patients.

Although non-haematological cancers seem to have less reliance on BCL-2 and related proteins, a study by Jeffrey Engelman and colleagues has identified the potential value of navitoclax for treating some epithelial cancers. KRAS is mutated in various cancers, but targeted therapeutics to exploit this mutation - such as blocking downstream signalling through MEK inhibition - have shown limited efficacy. The authors carried out a short hairpin RNA (shRNA) screen for gene knockdowns that sensitized two KRAS-mutant cell lines to a MEK inhibitor (selumetinib). They identified BCL- $\mathrm{X}_{\mathrm{L}}$ as the most potent hit, and they confirmed this pharmacologically using a combination of selumetinib and navitoclax. Follow-up biochemical studies indicated a mechanism whereby MEK inhibition causes upregulation of pro-apoptotic BIM, which is only a weak inducer of apoptosis unless the BIM-BCL-X interaction is disrupted by navitoclax.

From a panel of cancer cell lines, the cells that responded to this combination were KRAS-mutant cells of various tissue types that had an epithelial (rather than a mesenchymal) gene expression signature, which could potentially guide future patient stratification.

This combination also synergized to induce regression of mouse xenografts of human KRAS-mutant colorectal cancer cells, and tumour regression in an orthotopic mouse model of Kras-driven lung cancer. Although limited toxicity was reported in these animal studies, it is not yet known whether thrombocytopenia induced by navitoclax will hinder the clinical adoption of this combination.

For both approaches reported in these studies, it will be interesting to fully dissect the tumour genotypes and phenotypes that are predictive of a response, and to assess the potential for their long-term control of cancer.

Darren J. Burgess

ORIGINAL RESEARCH PAPERS Souers, A. J. et al. ABT-199, a potent and selective BCL-2 inhibitor, achieves antitumor activity while sparing platelets. Nature Med. 6 Jan 2013 (doi:10.1038/ $\mathrm{nm} .3048)$ |Corcoran, R. B. et al. Synthetic lethal interaction of combined BCL-XL and MEK inhibition promotes tumor regressions in KRAS mutant cancer models. Cancer Cell. 11 Dec 2012 (doi:10.1016/j.ccr.2012.11.007) 